\title{
An Evaluation of Intubation-Associated Vocal Cord Dysfunction after Cardiovascular Surgery in a Single Center
}

\section{OHiroki Taenaka, Sho Carl Shibata, Yuji Fujino \\ Department of Anaesthesiology and Intensive Care Medicine, Osaka University Graduate School of Medicine}

\section{Background}

- Vocal cord paralysis (VCP) is a rare but well documented complication of thoracic surgery. ${ }^{12}$

- VCP may cause aspiration and recurrent pneumonia in critically ill patients. ${ }^{3}$ Extubation failure and re-intubation have been associated with increased morbidity and mortality. ${ }^{4}$

- Although the relationship of risk factors between the frequency of VCP has already been reported, their effect on the severity of VCP has been less well studied. Furthermore, the frequency and severity of VCP in high-risk patients undergoing implantable ventricular assist device (VAD) surgery or in newer less invasive procedures such as endovascular debranching repair is unknown.

- The aim of our study was to evaluate the frequency and severity of VCP after cardiovascular surgery in order to update and identify possible risk factors for VCP.

\section{Methods}

- After obtaining institutional ethical review board approval, we performed a retrospective record based review of all adult patients who underwent cardiovascular surgery at our center between January 2013 and August 2015.

- The primary outcome was the frequency and severity of VCP.

- The severity of VCP was classified into following categories; Mild (cases with dysphonia with remission within 6 months), Moderate (partial to no recovery of VCP symptoms within 6 months), and Severe (cases with airway obstruction after extubation which required re-intubation).

- The risk factors such as sex, age, height, weight, BMI, smoking history, hypertension, diabetes mellitus, NYHA classification, left ventricular ejection fraction, intubation period, tube diameter, depth of intubation, intubation provider, surgical procedure, operation period, ITA harvest, and hypothermia were included in the analysis.

\section{Results}

Table 1. Patient characteristics

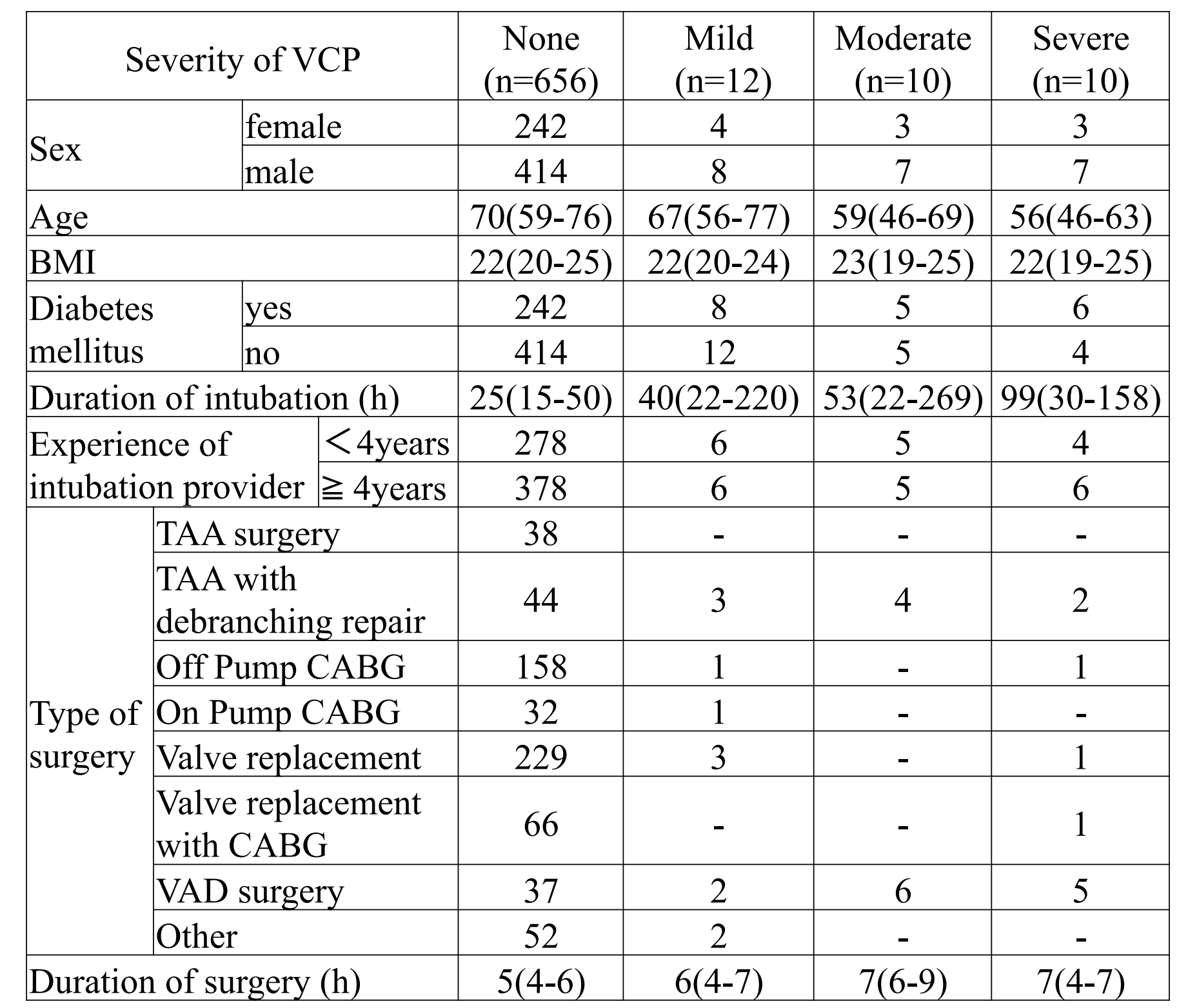

- We included a total of 756 patients during our 2 year study period. After excluding patients who did not meet our criteria 688 patients were included in our final analysis.

1. Hamdan A et al. Eur J of Cardio-thorac Surg 2001; 21:671-674

2. Domino KB et al. Anaesthesiology 1999; 91:1703-11

3. Pluijims W et al. Critical Care 2015; 19:295

4. Epstein SK et al. Am J Respir Crit Care Med 1998; 158:489-493
Table 2. The incidence and severity of VCP

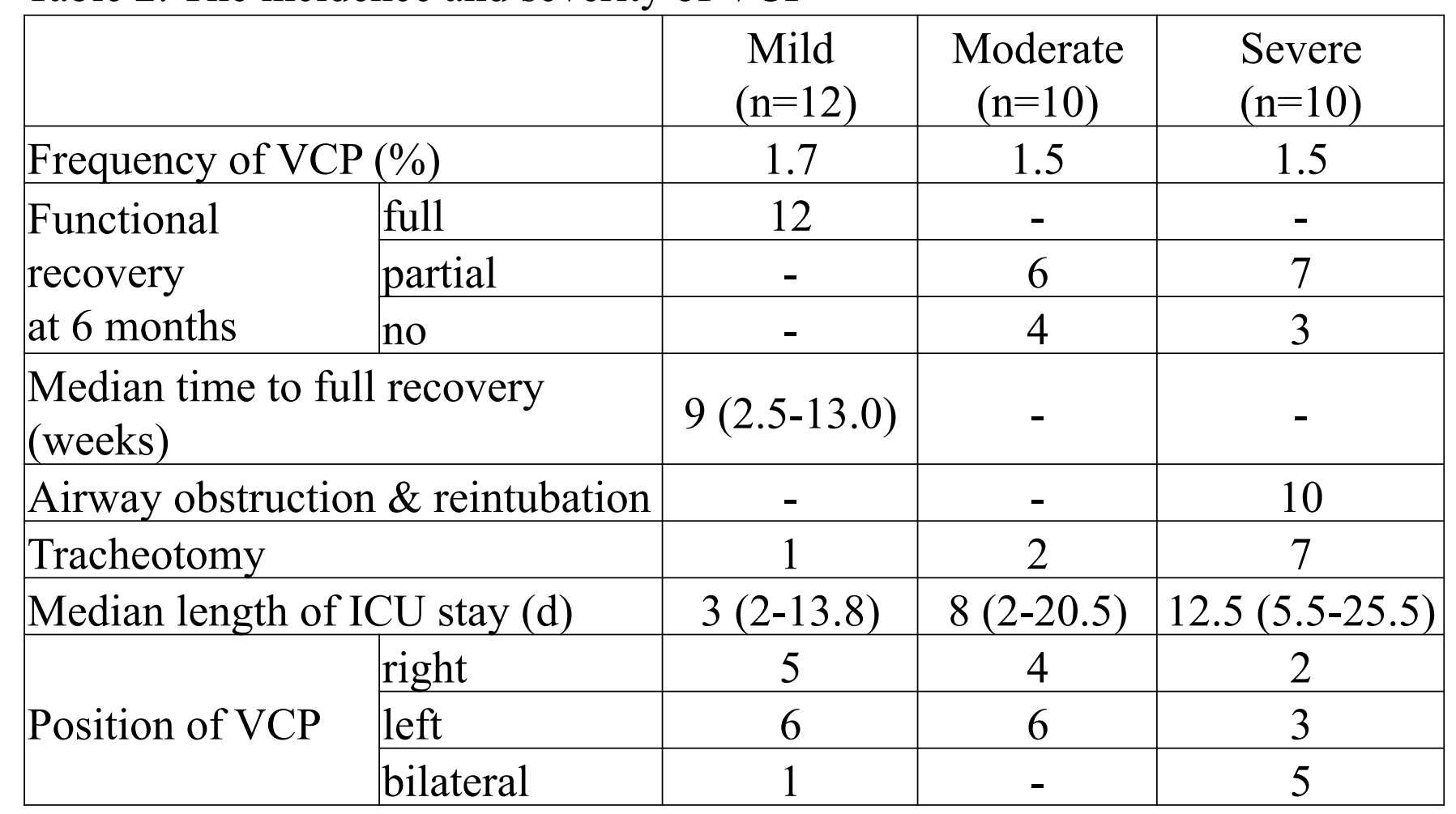

- VCP occurred in $32(4.65 \%)$ cases, with 12 mild (1.7\%), 10 moderate $(1.5 \%)$, and 10 severe $(1.5 \%)$ cases.

- The median recovery time was 9 weeks in the mild VCP group.

- More than half the patients in the moderate and severe groups had partial recovery of VCP within 6 months but 4 patients from the moderate and 3 patients from the severe group had permanent VCP.

- The types of surgery involved with permanent VCP were VAD implantation (4 cases), ascending aortic surgery with debranching repair ( 2 cases), and valve replacement surgery with CABG (1 case).

- Of the 10 cases of severe VCP, all patients were re-intubated and of which 7 patients later required tracheostomy.

Table 3. The risk factors of VCP (multivariate analysis)

\begin{tabular}{|c|c|c|c|}
\hline \\
\hline & Odds ratio & $P$ value & $95 \% \mathrm{CI}$ \\
\hline Female & 1.031 & 0.909 & $0.600-1.730$ \\
\hline Age (per decade) & 0.8 & 0.201 & $0.566-1.149$ \\
\hline BMI & 0.952 & 0.448 & $0.833-1.079$ \\
\hline Hypertension & 0.7 & 0.117 & $0.447-1.087$ \\
\hline Diabetes mellitus & 1.878 & 0.009 & $1.181-3.047$ \\
\hline $\mathrm{NYHA} \geqq 3$ & 1.891 & 0.048 & $1.000-3.656$ \\
\hline EF (per \%) & 1 & 0.995 & $0.970-1.031$ \\
\hline Intubation duration (per h) & 1.118 & 0.027 & $1.011-1.254$ \\
\hline Outer diameter $(>10.8 \mathrm{~mm})$ & 3.331 & 0.015 & $1.194-8.594$ \\
\hline Depth of intubation tube (per $\mathrm{cm})$ & 0.964 & 0.795 & $0.741-1.281$ \\
\hline Experience of intubation provider $\geqq 4 \mathrm{yr}$ & 0.929 & 0.728 & $0.611-1.421$ \\
\hline $\begin{array}{l}\text { Ascending aortic arch surgery } \\
\text { with debranching repair }\end{array}$ & 12.06 & $<0.001$ & $3.888-48.842$ \\
\hline Off pump CABG & 0.861 & 0.86 & $0.178-4.672$ \\
\hline Type of On pump CABG & 1.266 & 0.795 & $0.188-7.033$ \\
\hline surgery Valve replacement & 1.236 & 0.678 & $0.481-3.783$ \\
\hline Valve replacement with CABG & 0.878 & 0.879 & $0.138-4.360$ \\
\hline VAD & 2.755 & 0.03 & $1.185-9.079$ \\
\hline Duration of surgery (per h) & 1.074 & 0.503 & $0.871-1.315$ \\
\hline ITA harvest & 1.593 & 0.497 & $0.453-5.820$ \\
\hline Deep hypothermic cardiac arrest & 0.595 & 0.179 & $0.269-1.247$ \\
\hline
\end{tabular}

- Diabetes mellitus (OR $1.88 \mathrm{p}<0.01$ ), intubation period (OR per 24h 1.12 $\mathrm{p}=0.03$ ), endotracheal tube with a larger outer diameter larger than $10.8 \mathrm{~mm}$ (OR $3.33 \mathrm{p}=0.02$ ), ascending aortic arch surgery with debranching repair (OR 12.06, $\mathrm{p}<0.01$ ), and VAD implantation surgery (OR 2.76, $\mathrm{p}=0.03$ ) remained independent predictors for VCP after cardiovascular surgery.

- Other factors were not significant factors.

\section{Conclusion}

We should pay attention to intubation related VCP and perioperative respiratory complications in patients with diabetes mellitus, large endotracheal tube size, ascending aortic arch surgery with debranching repair, VAD implantation, and prolonged mechanical ventilation periods. 